\title{
Efficacy of chest expansion resistance exercise on respiratory function, trunk control and dynamic balance in patients with chronic stroke: A Comparative study.
}

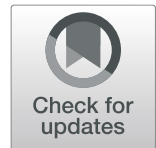

\author{
Shruti Prabhakaran Nair ${ }^{1^{*}}$ (D, Shailesh Satyanarayana Gardas² and Rukaiya Mithaiwala ${ }^{3}$
}

\begin{abstract}
Background: Motor impairments caused by stroke result in impaired diaphragmatic and respiratory muscle function, changes in thoracic biomechanics on the hemiparetic side ultimately resulting in decreased efficiency of lung ventilation. This study aimed to examine the efficacy of chest expansion resistance exercise (CERE) on respiratory function, trunk control ability, and balance in patients with chronic stoke. Following a purposive sampling, thirty-five patients with chronic stroke were randomly allocated into two groups, i.e., the experimental group receiving CERE with conventional therapy and the control group receiving conventional therapy alone. Both the groups received therapy four times per week for a period of four weeks (total 16 sessions). Following assessments were done before and after treatment in both the groups: chest expansion ( axillary, nipple, xiphisternal levels) using measure tape, respiratory muscle strength using micro-respiratory pressure meter, trunk control using the Trunk Impairment Scale, and balance using mini-Balance Evaluation Systems Test.

Results: Both groups had 17 participants each $(n=34$, drop-outs $=1$ ) consisting of 12 males and 5 females having a mean age of $56.5 \pm 12.98$ years and $59.7 \pm 10.2$ years, respectively. Intra-group analysis showed a statistically significant increase in mean values of chest expansion, respiratory muscle strength, trunk control ability, and balance in the experimental group whereas the control group showed improvement only in trunk control ability and balance. Inter-group comparison revealed a better improvement in all the outcome variables in experimental group compared to the control group.
\end{abstract}

Conclusions: Based on these results, this study proved that CERE was more effective in improving respiratory function, trunk control, and balance in patients with chronic stroke.

Keywords: CERE, Stroke rehabilitation, Respiratory function, Chronic stroke

\footnotetext{
* Correspondence: shrutinair2008@gmail.com

Presentation at a meeting: Trinity 2018 - Paper Presentation

Organization: Lokmanya Tilak Municipal General Hospital, Mumbai, India

'Department of Cardiovascular \& Pulmonary Physiotherapy, Mahatma Gandhi

Mission's College of Physiotherapy, Vashi, Navi, Mumbai 400705, India

Full list of author information is available at the end of the article
}

\section{Springer Open}

(- The Author(s). 2021 Open Access This article is licensed under a Creative Commons Attribution 4.0 International License, which permits use, sharing, adaptation, distribution and reproduction in any medium or format, as long as you give appropriate credit to the original author(s) and the source, provide a link to the Creative Commons licence, and indicate if changes were made. The images or other third party material in this article are included in the article's Creative Commons licence, unless indicated otherwise in a credit line to the material. If material is not included in the article's Creative Commons licence and your intended use is not permitted by statutory regulation or exceeds the permitted use, you will need to obtain permission directly from the copyright holder. To view a copy of this licence, visit http://creativecommons.org/licenses/by/4.0/. 


\section{Background}

According to World Health Organization, stroke is defined as a clinical syndrome consisting of rapidly developing clinical signs of focal disturbance of cerebral function lasting more than $24 \mathrm{~h}$ or leading to death with no apparent cause other than a vascular origin [1]. It is one of the main causes of death and disability worldwide with a very high incidence and prevalence rate [2]. During the past two decades, prevalence of stroke in different parts of India ranged from 44.29 to $559 / 100,000$ persons [3].

Motor impairment caused by stroke is one of the major deficits resulting in loss or limitation of motor control [4]. Previously, studies have demonstrated that stroke not only causes impairments in the peripheral muscles but also affects the respiratory muscle strength [5]. Hemiparesis being a common clinical presentation of stroke compromises the motor control necessary for maintaining synergy of respiratory muscles. Impaired function of the diaphragm and respiratory muscles on the paretic half of the body leads to asymmetric respiratory chest movements and changes in the respiratory biomechanics which eventually results in decrease in efficiency of lung ventilation [6]. Additionally, tonal abnormalities in the muscles of the chest wall and abdomen along with motor impairments cause a restrictive pattern of respiratory involvement [7]. Decreased maximal inspiratory pressures [8], muscle disuse of the hemiparetic side including the diaphragm, impaired rib cage mobility [6], along with impaired postural control, and truncal mal-alignment $[9,10]$ are commonly seen in chronic stroke patients. These impairments result in activity limitation thereby affecting their cardiopulmonary function and reducing the energy efficiency of the body [11].

Literature supports a strong relationship between trunk and respiratory muscle control [12]. Good sensorimotor ability of the trunk is an important factor for proximal stabilization, thereby playing an important role in balance, transfers, and functional independence after stroke $[9,13]$. Therefore, implementing respiratory interventions could potentially influence trunk control and also reduce the mortality and morbidity related to respiratory complications [14].

Traditionally, neuro-rehabilitation programs for stroke are based on theories of neuroplasticity and motor recovery focusing on balance and gait impairment. Goal settings to evaluate and enhance cardiopulmonary functions are scarcely prioritized. A study comparing the effectiveness of combined respiratory muscle training has shown positive effects on both respiratory function and expiratory muscle activity in stroke patients [15]. Another systematic review on patients with stroke has reported effectiveness of respiratory muscle training in improving respiratory muscle strength [16]. The chest expansion resistance exercise (CERE) is an exercise based on a proprioceptive neuromuscular facilitation (PNF) breathing exercise [17]. A recent study by Lucyna [7] found that a single application of respiratory stimulation using PNF significantly improved the lung function. Evidence supports effectiveness of CERE to improve stroke patient's walk distance, trunk control, degree of chest expansion, and forced expiratory volume in 1 s (FEV1) [11, 17].

So far, respiratory interventions in stroke patients have focused largely on its effectiveness on respiratory function. Moreover, CERE as an intervention has not been widely explored. There has been a dearth in literature studying the effect of CERE on respiratory muscles that could possibly have an effect not only on the respiratory functions but also on trunk control and balance in these patients. Hence, we aimed to investigate the effects of CERE on respiratory muscle strength, chest expansion, trunk control and balance in patients with chronic stroke.

\section{Methods}

This was an experimental study following a purposive sampling conducted in a physiotherapy rehabilitation outpatient department (OPD) of a tertiary care hospital. After seeking ethical approval from the Institutional Review Board (reference no. MGM/COP/IRRC/124/2018), the study purpose was explained to the participants and their written informed consent was sought.

A total of thirty-five patients diagnosed with stroke on the basis of computed tomography (CT) or magnetic resonance imaging findings (MRI) were selected as participants. The inclusion criteria adopted were stroke duration not shorter than 6 months, Mini-Mental State Examination score $<24$, and an ability to walk $10 \mathrm{~m}$ independently (with/without walking aid). Participants with a history of acute illness, respiratory disorders, other neurological conditions, acute rib fractures, the ones who had undergone respiratory rehabilitation for stroke, and the ones who refused to participate were excluded from the study.

The study participants were randomly allocated into two groups, i.e., the experimental and control group containing eighteen participants each using a chit method. The experimental group received chest expansion resistance exercises along with conventional neurorehabilitation four times per week for a period of four weeks (16 sessions). The control group received conventional neuro-rehabilitation program for the same number of sessions.

Pre- and post-assessment in both the groups included (A) chest expansion using measure tape, (B) respiratory muscle strength using micro-respiratory pressure meter, (C) trunk control using the Trunk Impairment Scale, 
and (D) balance using the Mini-Balance Evaluation Systems Test (BESTest).

\section{Outcome measures Chest expansion}

Chest expansion was measured using a non-stretchable inch tape at three different levels-axillary level (2nd intercostal space), nipple level (4th intercostal space), and xiphoid process level $[18,19]$. Participants were made to sit on a stool with the hands resting on the side of the trunk. Tape was wound around the chest wall at above mentioned levels and participants were asked to perform complete exhalation through the mouth and then take a maximal deep inspiration through their nose. The difference between the full expiration and full inspiration was noted (unit in inches). Three trials were taken at each level and average of three readings was noted.

\section{Respiratory muscle strength}

Respiratory muscle strength was recorded using a noninvasive micro-RPM (respiratory pressure meter) (manufacturer, Care Fusion Respiratory, 22745 Savi Ranch Pkwy, Yorba Linda, CA, USA). Micro-RPM is known to be a reliable, non-invasive measurement tool with good clinical utility [20].

Maximal inspiratory pressure (MIP) indicates inspiratory muscle strength whereas maximal expiratory pressure (MEP) indicates expiratory muscle strength. Respiratory pressures were assessed with participants in comfortable sitting position. They were instructed to insert the mouthpiece into the mouth, ensuring that the flange was positioned over the gums and inside the lips while the "bite blocks" were between the teeth.

Recording of MEP After inhaling to their total lung capacity, participants were asked to perform a forced exhalation with as much effort as possible (minimum $2 \mathrm{~s}$ ). The reading displayed MEP in centimeters of water $(\mathrm{cm}$ $\mathrm{H}_{2} \mathrm{O}$ ).

Recording of MIP The participants were instructed to exhale to residual volume and then perform a forced inhalation through the mouthpiece with as much effort as possible for at least $2 \mathrm{~s}$. The reading displayed MIP in centimeters of water $\left(\mathrm{cm} \mathrm{H}_{2} \mathrm{O}\right)$.

The test was repeated thrice and the best value was documented.

\section{Trunk control}

Trunk control was evaluated using the Trunk Impairment Scale (TIS). The TIS contains 17 sub-items in three categories: static sitting balance, dynamic sitting balance, and coordination. This tool offers high reliability and validity and is used to evaluate the degree of trunk motor impairment after stroke [21]. The highest possible TIS score is 23 points, with $0-7$ possible points for static sitting balance, $0-10$ points for dynamic sitting balance, and 0-6 points for coordination.

\section{Balance}

Balance was evaluated using mini-BESTest.

The Mini-BESTest is a 14-item balance scale that measures dynamic balance specifically anticipatory transitions, postural responses, and sensory orientation while standing on a compliant or inclined base of support, and dynamic stability during gait. Each task is rated on a 2point ordinal scale from 0 to 2; a score of 0 indicating that a person is unable to perform the task, score of 2 indicates normal performance. The maximum score for this scale is 28 . High intra-rater and inter-rater reliability and validity of this tool have been reported in stroke patients [22, 23].

\section{Intervention program \\ Experimental group}

This group received chest expansion resistance exercise (CERE) along with conventional neuro-rehabilitation protocol.

CERE is based on the concept of proprioceptive neuromuscular facilitation (PNF) which facilitates respiration. Participants were made to lie on their side with the affected side up. The therapist palpated the anterior and posterior aspects of the thorax along the costal margins as if embracing the rib-cage. The therapist then instructed the participant to exhale completely and applied a stretch at end expiration in the medial and caudal direction. This was followed by application of continuous resistance in the same direction throughout the next inspiratory cycle thereby allowing him/her to perform an active respiratory exercise. The amount of resistance was such that it caused responses in all ranges of motion but not to such a degree that movements were suppressed. Each exercise was performed 15 times per set for a total of 10 sets. Rest pause of $30 \mathrm{~s}$ was given in between the sets [17].

In addition to CERE, participants also received conventional neurological rehabilitation protocol which included the following:

1. Sustained stretching of all spastic muscles-3 sets with $30 \mathrm{~s}$ hold

2. Antigravity/weight bearing postures such as kneeling and quadruped as tolerated by patient with necessary assistance

3. Reach outs with unaffected hand in weight bearing in sitting and quadruped

4. PNF pattern of bilateral upper and lower extremity 
5. Strengthening of weak antagonist muscles with manual resistance

6. Balance training with perturbations in sitting and standing position

7. Functional training and transfers

8. Gait training, forward walking, backward walking, and lunges

\section{Control group}

This group received only conventional neurological rehabilitation protocol.

Both the groups received intervention four times per week for a period of 4 weeks (16 sessions).

\section{Statistical analysis}

Data obtained was coded, tabulated using Microsoft Excel and results were analyzed using Statistical Package for Social Science software (SPSS version 20). Normality of the data distribution was assessed using Shapiro-Wilk test which revealed that the data was normally distributed. Hence, paired $t$ test was used for intra-group comparison and independent $t$ test was used for intergroup comparison. Data was presented as mean and standard deviation. The statistical significance level $\alpha$ was set to 0.05 .

\section{Results}

Thirty-five patients with chronic stroke were included in this study. One participant from experimental group dropped out in the second week because he discontinued coming for therapy to the OPD and had to be excluded from the study. Thus, data was analyzed for 34 participants (17 in experimental and 17 in control group) who completed all assessments and training (Fig. 1).

The mean age of participants in the experimental group was $56.5 \pm 12.98$ years and in the control group was $59.7 \pm 10.2$ years. Both the groups comprised of 12 males and 5 females.

The baseline characteristics of the groups are presented in Table 1. Both groups were homogenous in terms of the studied parameters $(p>0.05)$. Tables 2 and 3 shows intra-

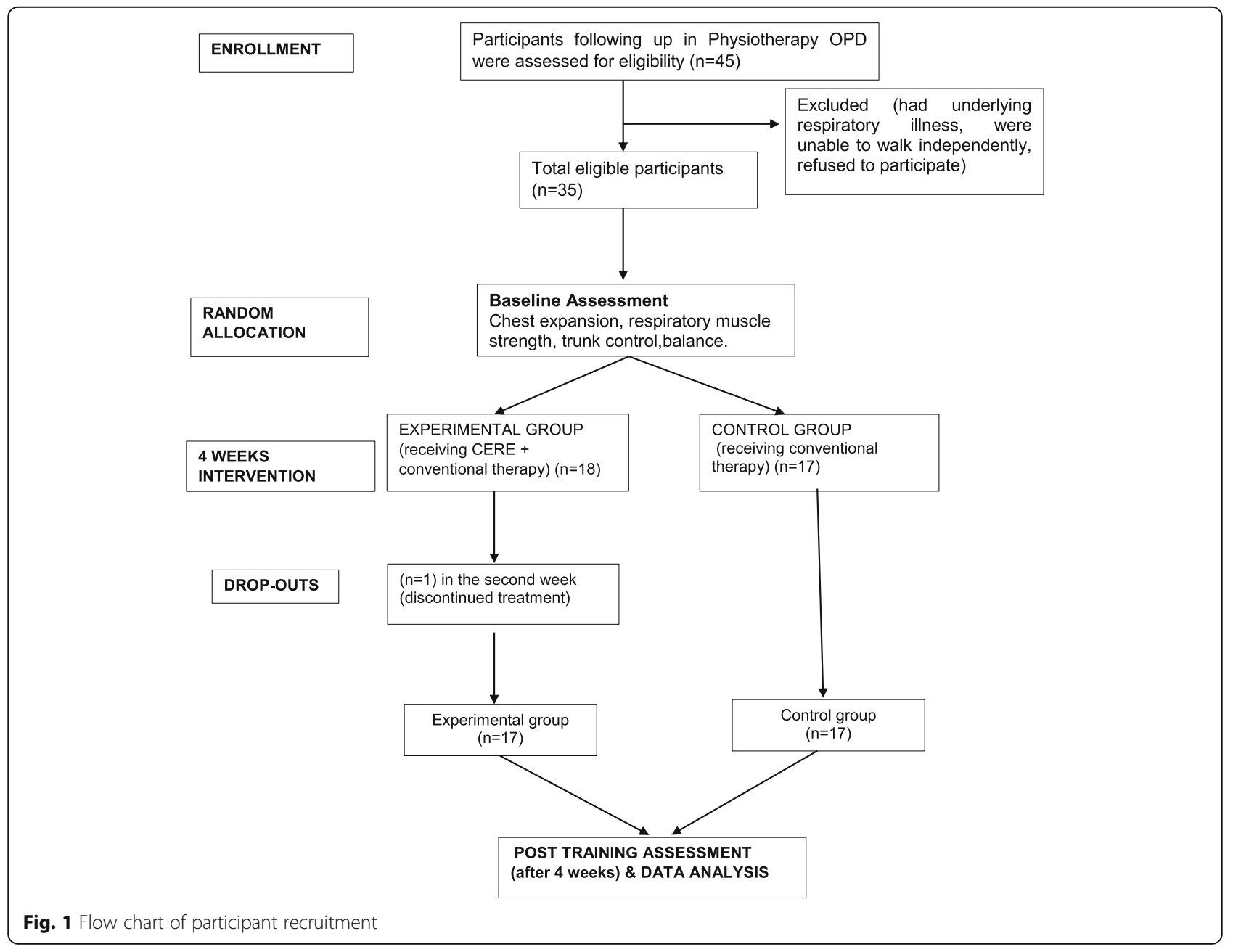


Table 1 Baseline characteristics of participants allocated to the experimental and control group

\begin{tabular}{|c|c|c|c|}
\hline Variables & Experimental group $(n=17)$ & Control group $(n=17)$ & $p$ value \\
\hline Age (years) & $56.5(12.98)$ & $59.7(10.2)$ & 0.22 \\
\hline \multicolumn{4}{|c|}{ Chest expansion (in inches) } \\
\hline Axillary & $0.76(0.15)$ & $0.77(0.13)$ & 0.357 \\
\hline Nipple & $0.91(0.14)$ & $0.92(0.18)$ & 0.389 \\
\hline Xiphisternal & $0.63(0.13)$ & $0.60(0.19)$ & 0.374 \\
\hline \multicolumn{4}{|c|}{ Respiratory muscle strength (in $\mathrm{cm} \mathrm{H}_{2} \mathrm{O}$ ) } \\
\hline MIP & $33.56(6.38)$ & $35.75(9.12)$ & 0.425 \\
\hline MEP & $45(9.04)$ & $43.68(9.51)$ & 0.280 \\
\hline \multicolumn{4}{|l|}{ Balance } \\
\hline Mini-BESTest score & $10.56(3.14)$ & $10.43(4.07)$ & 0.151 \\
\hline \multicolumn{4}{|l|}{ Trunk control } \\
\hline TIS score & $10.06(2.54)$ & $10.43(4.07)$ & 0.276 \\
\hline
\end{tabular}

Values expressed as mean (standard deviation)

MIP maximal inspiratory pressure, MEP maximal expiratory pressure, TIS Trunk Impairment Scale

and inter-group analysis respectively of different parameters like chest expansion (all three levels), respiratory muscle strength (MIP, MEP), balance (score), and trunk control (TIS score) in both the groups.

Within group analysis showed a statistically significant increase in mean values of chest expansion (axillary, nipple, and xiphisternal levels), respiratory muscle strength (MIP, MEP), balance (mini-BESTest score), and trunk control (TIS score) in the experimental group whereas the control group showed improvement only in balance and trunk control ability as shown in Table 2 .

Between-group comparison showed a statistical significant change as seen by increased mean difference values of chest expansion (all three levels), respiratory muscle strength (MIP, MEP), balance (mini-BESTest score), and trunk control (TIS score) in experimental group compared to the Control group (Table 3).

\section{Discussion}

The present study aimed to investigate the effect of CERE on chest expansion, respiratory muscle strength, balance, and trunk control in patients with chronic stroke. Results showed significant improvement in chest expansion, respiratory muscle strength, balance, and trunk control ability in participants receiving CERE along with conventional neuro-rehabilitation, whereas control group participants showed improvement only in balance and trunk control ability.

CERE along with conventional neuro-rehabilitation brought about a $20 \%$ improvement in MIP and $25 \%$

Table 2 Intra-group comparison of chest expansion, respiratory muscle strength, mini-BESTest score, and TIS in the experimental and control group

\begin{tabular}{|c|c|c|c|c|c|c|}
\hline \multirow[t]{2}{*}{ Variables } & \multicolumn{3}{|c|}{ Experimental group $(n=17)$} & \multicolumn{3}{|c|}{ Control group $(n=17)$} \\
\hline & Pre & Post & $p$ value & Pre & Post & $p$ value \\
\hline \multicolumn{7}{|c|}{ Chest expansion (in inches) } \\
\hline Axillary & $0.76(0.15)$ & $0.9(0.12)$ & $0.003^{\#}$ & $0.77(0.13)$ & $0.78(0.14)$ & 0.564 \\
\hline Nipple & $0.91(0.14)$ & $1.11(0.14)$ & $0.001^{*}$ & $0.92(0.18)$ & $0.98(0.2)$ & 0.083 \\
\hline Xiphisternal & $0.63(0.13)$ & $0.8(0.16)$ & $0.001^{*}$ & $0.60(0.19)$ & $0.62(0.21)$ & 0.083 \\
\hline \multicolumn{7}{|c|}{ Respiratory muscle strength (in $\mathrm{cm} \mathrm{H}_{2} \mathrm{O}$ ) } \\
\hline MIP & $33.56(6.38)$ & $41.75(7.34)$ & $0.001^{*}$ & $35.75(9.12)$ & $35.87(8.97)$ & 0.480 \\
\hline MEP & $45(9.04)$ & $60.31(8.62)$ & $0.001^{*}$ & $43.68(9.51)$ & $44.18(9.15)$ & 0.075 \\
\hline \multicolumn{7}{|l|}{ Balance } \\
\hline Mini-BESTest score & $10.56(3.14)$ & 9.75 (2.20) & $0.000^{*}$ & $10.43(4.07)$ & 17.18 (3.69) & $0.001^{*}$ \\
\hline \multicolumn{7}{|l|}{ Trunk control } \\
\hline TIS score & $10.06(2.54)$ & 17.31 (1.92) & $0.000^{*}$ & 11.06 (2.79) & $15.43(1.82)$ & $0.001^{*}$ \\
\hline
\end{tabular}

Values expressed as mean (standard deviation)

${ }^{*} p<0.001$; significant differences between pre- and post-test in both the experimental and control group

${ }^{\#} p<0.01$; significant differences between pre- and post-test in both the experimental and control group 
Table 3 Inter-group comparison of chest expansion, respiratory muscle strength, mini-BESTest score, and TIS in the experimental and control group

\begin{tabular}{|c|c|c|c|}
\hline \multirow[t]{2}{*}{ Variables } & Experimental group $(n=17)$ & Control group $(n=17)$ & $p$ \\
\hline & \multicolumn{2}{|l|}{ Mean difference (Standard Deviation) } & \\
\hline \multicolumn{4}{|l|}{ Chest expansion } \\
\hline Axillary & $0.13(0.07)$ & $0.006(0.04)$ & $0.000^{*}$ \\
\hline Nipple & $0.2(0.06)$ & $0.025(0.04)$ & $0.000^{*}$ \\
\hline Xiphisternal & $0.16(0.07)$ & $0.018(0.04)$ & $0.000^{*}$ \\
\hline \multicolumn{4}{|c|}{ Respiratory muscle strength } \\
\hline MIP & $8.25(2.48)$ & $0.125(0.71)$ & $0.000^{*}$ \\
\hline MEP & $15.31(3.80)$ & $0.625(1.08)$ & $0.000^{*}$ \\
\hline \multicolumn{4}{|l|}{ Balance } \\
\hline Mini-BESTest score & $9.18(1.68)$ & $6.75(1.34)$ & $0.003^{\#}$ \\
\hline \multicolumn{4}{|l|}{ Trunk control } \\
\hline TIS score & $7.25(1.5)$ & $4.37(1.58)$ & $0.000^{*}$ \\
\hline
\end{tabular}

${ }^{*} p<0.001$; significant differences between the experimental and control group

$\# p<0.01$; significant differences between the experimental and control group

improvement in MEP whereas control group participants receiving only conventional therapy did not show any significant improvement. Likewise, chest expansion values improved by $15 \%$ (axillary), 18\% (nipple), and 21\% (xiphisternal) in the experimental group whereas the control group showed minimal improvement. CERE is known to work on the principle of PNF technique [17]. The physiological mechanism of PNF is based on the stretch reflex which resists the change in muscle length thereby facilitating contraction in the stretched muscle fiber via its muscle spindle [24]. Direct resistance applied by the therapist during inspiration could have increased the intra thoracic and intra-abdominal pressures thereby resulting in improved activity of the diaphragm and the accessory muscles of inspiration [24]. This likely could have caused an improvement in respiratory muscle strength as seen in this study. Improved strength in the inspiratory muscles because of the resistance offered during CERE along with reduced chest wall stiffness due to the stretch applied at end expiration could have possibly contributed to enhanced expansibility of the thorax as proven in other studies $[25,26]$ Strength and mobility of respiratory muscles, elasticity of surrounding soft tissues determines the capacity of thorax which has a direct effect on the chest wall movement [17, 27]. This could have been further assisted by the thrust given at the end expiration while applying CERE [28]. These findings were supported by Fernanda Lanza et al. who found that greater the inspiratory muscle strength, the greater the expansion of both the upper and the lower rib cage [29]. Kim et al. in their study on chronic stroke patients proved that CERE improved chest expansion attributing the changes to facilitation of respiration causing increased inflow of air into the lungs. However, pulmonary function test results (FEV1, FVC, vital capacity) did not show any significant changes [30]. In another study performed on elderly, Kim et al. documented a significant change in chest expansion and inspiratory muscles strength following 6 weeks of CERE wherein the elderly participants showed an improvement of $48 \%$ in chest expansion and 25\% in MIP values [17].

Trunk plays an important role as a central axis for functional movements of the extremities and also as proximal stabilizers in order to perform distal movements efficiently. Improved respiratory muscle strength facilitated by both tonic and phasic activities in the respiratory muscles promotes a central nervous system response of coordination between respiratory and postural mechanism thus bringing about good trunk control [31, 32]. Therefore, it is said that if proximal trunk control is enhanced, there will be improvements in the proactive and reactive posture control required for mobility and balance [33].

The present study examined the changes in balance and trunk control ability using the mini-BESTest scale and TIS respectively in stroke patients. Along with improvement in respiratory functions, CERE resulted in an improvement in both balance and trunk control far more significantly in the experimental group as compared to control. This improvement could be attributed to positive influence of respiratory functions on trunk control as has been proven in previous studies [34]. Also, improvement in strength of abdominals and internal intercostals as seen by increase in MEP values post-CERE intervention could have been responsible for better trunk stabilization [31]. Our findings are supported by study conducted by Jandt et al. wherein TIS showed a significant relationship with PEFR and MEP 
[12]. Another study by Hodges et al. confirmed that contraction of the respiratory muscles is related to trunk control, and the activity of the motor neuron is organized in such a way that it contributes for both maintenance of posture and breathing during a task requiring repetitive changes of trunkal posture [32]. Chang et al. [35] and Song et al. [11] in their respective studies have also pointed out the role of respiratory muscles to bring about positive changes in physical efficiency and body stability thereby influencing postural control.

The findings seen in this study are corroborated by evidence reported in previous literature. Study by Lee et al. [36] confirmed that 4 weeks of respiratory exercises using respiratory exercise equipment (Lung Boost Respiratory Trainer MD8000) as an intervention positively helped improve trunk control, trunk muscle activity, and pulmonary function in patients with chronic stroke. Kim et al. [37] reported that respiratory strength training program using a threshold Positive Expiratory Pressure (PEP) device was effective in stabilizing trunk control in stroke patients. Likewise, Jo et al. [15] reported that combining respiratory muscle training with conventional stroke rehabilitation was effective in stabilizing the trunk muscle activity of the rectus abdominis, external abdominal oblique, and internal abdominal oblique muscles in patients with chronic stroke, which was consistent with the results of this study.

Findings of this study could serve as a basis for the development and implementation of revised rehabilitation protocols incorporating respiratory interventions in the form of CERE while treating patients with stroke. These respiratory interventions have a potential to not only improve the cardiopulmonary functions, but also may help in improving the balance and trunk control ability thereby possibly facilitating functional performance of these patients.

There are certain limitations noted in this study. At first, it is difficult to generalize the current observations owing to limited number of participants from a single study set up. Therefore, a study with a larger sample size including participants from multiple clinical set ups could be carried out in the future. Secondly, this study investigated only the short-term effects of CERE without any follow-up assessment. Future prospective studies on a larger subset of stroke patients, with regular follow-up to ascertain how long the effect of training endures, would prove to be beneficial. Another potential limitation could be the use of tape method to assess chest expansion which is a crude way of testing thoracic mobility; more robust and standardized tools like three-dimensional motion analysis, respiratory plethysmography via induction, magnetometry, etc. could perhaps have provided better measurement of rib cage mobility.

\section{Conclusions}

This study confirmed that respiratory intervention in the form chest expansion resistance exercise given along with conventional neuro-rehabilitation improved chest expansion, respiratory muscle strength, trunk control, and balance in patients with chronic stroke. Therefore, emphasis on incorporation of respiratory exercises as a part of neuro-rehabilitation intervention should be considered a priority while planning rehabilitation goals for patients suffering from stroke.

\section{Abbreviations \\ CERE: Chest expansion resistance exercise; PNF: Proprioceptive Neuromuscular Facilitation; FEV1: Forced expiratory volume in $1 \mathrm{~s}$; mini BESTest: Mini-Balance Evaluation Systems Test; CE: Chest Expansion; RPM: Respiratory pressure meter; MIP: Maximal inspiratory pressure: MEP: Maximal expiratory pressure; TIS: Trunk Impairment Scale}

\section{Acknowledgements}

The authors acknowledge the patients for their participation and cooperation in the study.

\section{Authors' contributions}

SN and SG have contributed to the concept, study design, literature search, data analysis, manuscript preparation, editing, and final review. RM has contributed to the design, literature search, data collection, data analysis, and manuscript preparation. The authors have read and approved the final manuscript.

\section{Funding}

The authors received no financial support for the research, authorship, and/ or publication of this article.

\section{Availability of data and materials}

The datasets used and/or analyzed during the current study are available from the corresponding author on reasonable request.

\section{Declarations}

Ethics approval and consent to participate

The Institutional Research Review Committee, Mahatma Gandhi Mission's, College of Physiotherapy, Navi, Mumbai (reference no. MGM/COP/IRRC/124/ 2018), approved the study. The subjects participating in the study were given a detailed explanation of the purpose and the procedures of the study in the language best understood by them. Written consent was taken from each participant in the language understood by them before commencing the study.

\section{Consent for publication}

The participants consented to the use of their details for this study.

\section{Competing interests}

The authors declare that they have no competing interests.

\section{Author details}

'Department of Cardiovascular \& Pulmonary Physiotherapy, Mahatma Gandhi Mission's College of Physiotherapy, Vashi, Navi, Mumbai 400705, India. 2Department of Neuro Physiotherapy, Mahatma Gandhi Mission's College of Physiotherapy, Navi Mumbai, India. ${ }^{3}$ Mahatma Gandhi Mission's College of Physiotherapy, Navi Mumbai, India.

Received: 6 May 2021 Accepted: 1 August 2021

Published online: 08 December 2021

\section{References}

1. Mozaffarian D, Benjamin EJ, Go AS, Arnett DK, Blaha MJ, Cushman M, et al. Heart disease and stroke statistics-2016 update: a report from the American 
Heart Association. Circulation. 2016;133(4):e38-360. https://doi.org/10.1161/ ClR.0000000000000350

2. Feigin VL, Lawes CM, Bennett DA, Barker-Collo SL, Parag V. Worldwide stroke incidence and early case fatality reported in 56 population-based studies: a systematic review. Lancet Neurol. 2009;8(4):355-69. https://doi. org/10.1016/S1474-4422(09)70025-0

3. Kamalakannan S, Gudlavalleti ASV, Gudlavalleti VSM, Goenka S, Kuper H. Incidence \& prevalence of stroke in India: a systematic review. Indian J Med Res. 2017;146(2):175-85. https://doi.org/10.4103/ijmr.IJMR_516_15.

4. Arene $\mathrm{N}$, Hidler J. Understanding motor impairment in the paretic lower limb after a stroke: a review of the literature. Top Stroke Rehabil. 2009;16(5): 346-56. https://doi.org/10.1310/tsr1605-346.

5. Pollock RD, Rafferty GF, Moxham J, Kalra L. Respiratory muscle strength and training in stroke and neurology: a systematic review. Int J Stroke. 2013;8(2): 124-30. https://doi.org/10.1111/j.1747-4949.2012.00811.x.

6. Howard RS, Rudd AG, Wolfe CD, Williams AJ. Pathophysiological and clinical aspects of breathing after stroke. Postgrad Med J. 2001;77(913):700-2. https://doi.org/10.1136/pmj.77.913.700.

7. Ptaszkowska L, Ptaszkowski K, Halski T, Taradaj J, Dymarek R, PaprockaBorowicz M. Immediate effects of the respiratory stimulation on ventilation parameters in ischemic stroke survivors. Medicine. 2019:98(38):e17128.

8. Lanini B, Bianchi R, Romagnoli I, Coli C, Binazzi B, Gigliotti F, et al. Chest wall kinematics in patients with hemiplegia. Am J Respir Crit Care Med. 2003; 168(1):109-13

9. Tsuji T, Liu M, Hase K, Masakado Y, Chino N. Trunk muscles in persons with hemiparetic stroke evaluated with computed tomography. J Rehabil Med. 2003;35(4):184-8.

10. Oliveira CB, Medeiros IR, Frota NA, Greters MA, Conforto AB. Balance control in hemiparetic stroke patients: main tools for evaluation. J Rehabil Res Dev. 2008:45(8):1215-26. https://doi.org/10.1682/JRRD.2007.09.0150.

11. Song GB, Park FC. Effects of chest resistance exercise and chest expansion exercise on stroke patients' respiratory function and trunk control ability. J Phys Ther Sci. 2015;27(6):1655-8. https://doi.org/10.1589/jpts.27.1655.

12. Jandt SR, Caballero RM, Junior LA, Dias AS. Correlation between trunk control, respiratory muscle strength and spirometry in patients with stroke: an observational study. Physiother Res Int. 2011;16(4):218-24. https://doi. org/10.1002/pri.495.

13. Kim J-H, Lee S-M, Jeon S-H. Correlations among trunk impairment, functional performance, and muscle activity during forward reaching tasks in patients with chronic stroke. J Phys Ther Sci. 2015;27(9):2955-8.

14. Rochester CL, Mohsenin V. Respiratory complications of stroke. Semin Respir Crit Care Med. 2002;23(3):248-60. https://doi.org/10.1055/s-2002-33033.

15. Jo MR, Kim NS. Combined respiratory muscle training facilitates expiratory muscle activity in stroke patients. J Phys Ther Sci. 2017;29(29):1970-3. https://doi.org/10.1589/jpts.29.1970.

16. Menezes KK, Nascimento LR, Avelino PR, Alvarenga MTM, Teixeira-Salmela LF. Efficacy of interventions to improve respiratory function after stroke. Respir Care. 2018;63(7):920-33. https://doi.org/10.4187/respcare.06000.

17. Kim CB, Yang JM, Choi JD. The effects of chest expansion resistance exercise on chest expansion and maximal respiratory pressure in elderly with inspiratory muscle weakness. J Phys Ther Sci. 2015;27(4):1121-4. https://doi. org/10.1589/jpts.27.1121.

18. Bockenhauer SE, Chen H, Julliard KN, Weedon J. Measuring thoracic excursion: reliability of the cloth tape measure technique. J Am Osteopath Assoc. 2007:107(5):191-6.

19. Mohan V, Dzulkifli N, Justine M, Haron R, Joseph H, L., \& Rathinam, C. Intrarater reliability of chest expansion using cloth tape measure technique. Bangladesh Journal of Medical Science. 2012;11(4):307-11. https://doi.org/1 0.3329/bjms.v11i4.12602.

20. Dimitriadis Z, Kapreli E, Konstantinidou I, Oldham J, Strimpakos N. Test/retest reliability of maximum mouth pressure measurements with the Micro RPM in healthy volunteers. Respir Care. 2011;56(6):776-82. https://doi.org/10.41 87/respcare.00783.

21. Verheyden G, Nieuwboer A, Mertin J, Preger R, Kiekens C, de Weerdt W. The Trunk Impairment Scale: a new tool to measure motor impairment of the trunk after stroke. Clin Rehabil. 2004;18(3):326-34. https://doi.org/10.1191/02 69215504cr7330a.

22. Godi M, Franchignoni F, Caligari M, Giordano A, Turcato AM, Nardone A. Comparison of reliability, validity, and responsiveness of the mini-BESTest and Berg Balance Scale in patients with balance disorders. Phys Ther. 2013; 93(2):158-67. https://doi.org/10.2522/ptj.20120171.
23. Charlotte SL. Tsang, Lin-Rong Liao, Raymond C.K. Chung, Marco Y.C. Pang. Psychometric properties of the Mini-Balance Evaluation Systems Test (MiniBESTest) in community-dwelling individuals with chronic stroke. Phys Ther. 2013;93(8):1102-15.

24. Paulraj M, Shristhudhi SD, Anandbabu K. Effectiveness of PNF of respiration to improve the exercise capacity in patients with COPD: a pilot study. International Journal of World Research. 2017;1(35).

25. Minoguchi H, Shibuya M, Miyagawa T, Kokubu F, Yamada M. Tanaka H, et al; Cross-over comparison between respiratory muscle stretch gymnastics and inspiratory muscle training. Intern Med. 2002 Oct;41(10):805-12. https://doi. org/10.2169/internalmedicine.41.805.

26. Kim K, Fell DW, Lee JH. Feedback respiratory training to enhance chest expansion and pulmonary function in chronic stroke: a double-blind, randomized controlled study. J Phys Ther Sci. 2011;23(1):75-9. https://doi. org/10.1589/jpts.23.75.

27. Dietz B. International PNF basic course book. Gwangjoo Korea; 2006.

28. Pryor P. Physiotherapy for respiratory and cardiac problems.3rd edition. Churchill Livingtone; 2002.

29. Lanza FC, de Camargo AA, Archija LR, Selman JP, Malaguti C, Dal CS. Chest wall mobility is related to respiratory muscle strength and lung volumes in healthy subjects. Respir Care. 2013;58(12):2107-12. https://doi.org/10.4187/ respcare.02415.

30. Kim CB, Shin JH, Choi JD. The effect of chest expansion resistance exercise in chronic stroke patients: a randomized controlled trial. J Phys Ther Sci. 2015;27(2):451-3. https://doi.org/10.1589/jpts.27.451.

31. Sunders SW, Rath D, Hodges PW. Postural and respiratory activation on the trunk muscles changes with mode and speed of locomotion. Gait Posture. 2004;20(3):280-90. https://doi.org/10.1016/.gaitpost.2003.10.003.

32. Hodges PW, Heijnen I, Gandevia SC. Postural activity of the diaphragm is reduced in humans when respiratory demand increases. J Physiol. 2001; 537(Pt 3):999-1008. https://doi.org/10.1113/jphysiol.2001.012648.

33. An S-H, Park D-S. The effects of trunk exercise on mobility, balance and trunk control of stroke patients. J Korean Soc Phys Med. 2017;12(1):25-33. https://doi.org/10.13066/kspm.2017.12.1.25.

34. Santos R S'a, dos Dall'alba SCF, Forgiarini SGI, Rossato D, Dias AS, Junior F, et al. Relationship between pulmonary function, functional independence, and trunk control in patients with stroke. Arq Neuropsiquiatr. 2019;77(6): $387-92$.

35. Chang A, Paratz J, Rollston J. Ventilatory effects of neurophysiological facilitation and passive movement in patients with neurological injury. Aust J Physiother. 2002;48(4):305-10. https://doi.org/10.1016/S0004-9514(14)601 70-7.

36. Lee DK, Kim SH. The effect of respiratory exercise on trunk control, pulmonary function, and trunk muscle activity in chronic stroke patients. Phys Ther Sci. 2018;30(5):700-3. https://doi.org/10.1589/jpts.30.700.

37. Kim MH, Lee $\mathrm{WH}$, Yun MJ. The effects on respiratory strength training on respiratory function and trunk control in patient with stroke. J Korean Soc Phys Ther. 2012;24:340-7.

\section{Publisher's Note}

Springer Nature remains neutral with regard to jurisdictional claims in published maps and institutional affiliations.

\section{Submit your manuscript to a SpringerOpen ${ }^{\circ}$ journal and benefit from:}

- Convenient online submission

- Rigorous peer review

- Open access: articles freely available online

High visibility within the field

- Retaining the copyright to your article

Submit your next manuscript at $\boldsymbol{\nabla}$ springeropen.com 